\title{
Efficient Counting and Asymptotics of $k$-Noncrossing Tangled Diagrams
}

\author{
William Y. C. Chen ${ }^{1}$, Jing Qin ${ }^{2}$, Christian M. Reidys ${ }^{3}$ \\ Center for Combinatorics, LPMC-TJKLC \\ Nankai University, \\ Tianjin 300071, P. R. China \\ ${ }^{1}$ chen@nankai.edu.cn, ${ }^{2}$ qj@cfc.nankai.edu.cn, ${ }^{3}$ duck@santafe.edu \\ Doron Zeilberger \\ Mathematics Department \\ Rutgers University, New Brunswick, \\ Piscataway, NJ, USA \\ zeilberg@math.rutgers.edu
}

Submitted: Feb 25, 2008; Accepted: Mar 4, 2009; Published: Mar 13, 2009

Mathematics Subject Classification: 05A18

\begin{abstract}
In this paper, we enumerate $k$-noncrossing tangled-diagrams. A tangled-diagram is a labeled graph with vertices $1, \ldots, n$, having degree $\leq 2$, which are arranged in increasing order in a horizontal line. The arcs are drawn in the upper halfplane with a particular notion of crossings and nestings. Our main result is the asymptotic formula for the number of $k$-noncrossing tangled-diagrams $T_{k}(n) \sim$ $c_{k} n^{-\left((k-1)^{2}+(k-1) / 2\right)}\left(4(k-1)^{2}+2(k-1)+1\right)^{n}$ for some $c_{k}>0$.
\end{abstract}

\section{Tangled diagrams as molecules or walks}

In this paper we compute the numbers of $k$-noncrossing tangled diagrams and prove the asymptotic formula

$$
T_{k}(n) \sim c_{k} n^{-\left((k-1)^{2}+(k-1) / 2\right)}\left(4(k-1)^{2}+2(k-1)+1\right)^{n}, \quad c_{k}>0 .
$$

This article is accompanied by the Maple package TANGLE, which is available at http : //www.math.rutgers.edu/ zeilberg/mamarim/mamarimhtml/tangled.html. 


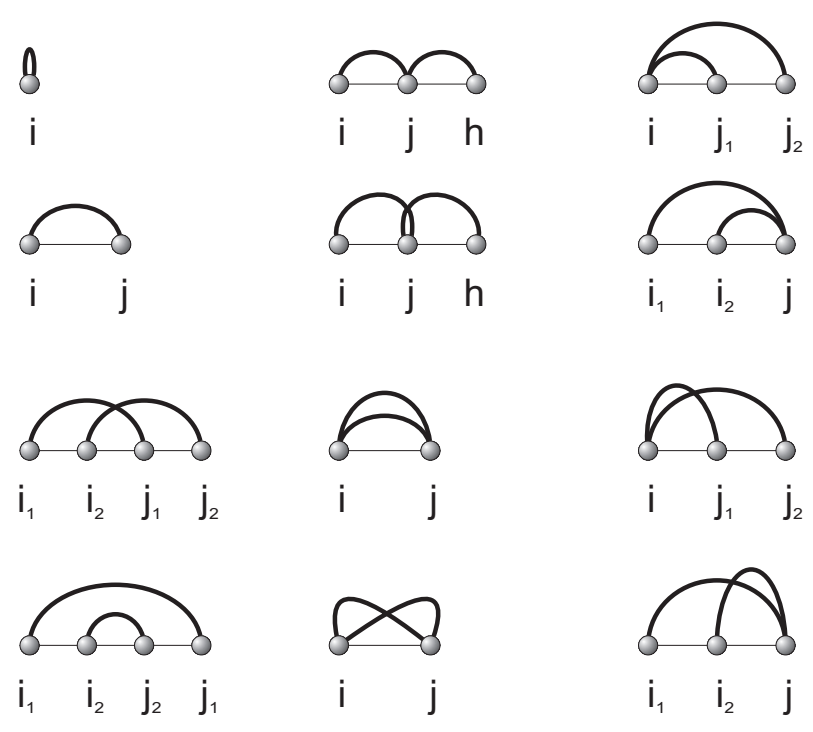

Figure 1: Arcs in tangled diagrams: a list of all possible arc-configurations.

$k$-noncrossing tangled diagrams are motivated by the studies of RNA molecules. They serve as combinatorial frames for searching molecular configurations and were recently studied [5] by the first three authors. Let us recall that a tangled diagram, or tangle, is a labeled graph over the vertex set $[n]=\{1, \ldots, n\}$, with vertices of degree at most two, drawn in increasing order in a horizontal line. Their arcs are drawn in the upper halfplane. In general, a tangled diagram has isolated points and its types of nonisolated vertices are given in Fig. 1. Tangled diagrams have possibly isolated points, for instance, the tangled diagram displayed in Fig. 1 has the isolated points 2 and 12.

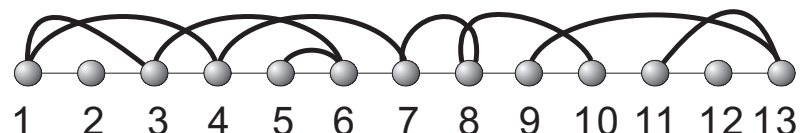

Figure 2: A tangled diagram over 13 vertices.

In order to describe the geometric crossings in tangled diagrams, we map a tangled diagram into a partial matching. This mapping is called inflation and intuitively "splits" each vertex of degree two, $j$, into two vertices $j$ and $j^{\prime}$ having degree one, see Fig. 3. Accordingly, a tangle with $\ell$ vertices of degree two is expanded into a diagram over $n+\ell$ vertices. Clearly, the inflation map has a unique inverse, obtained by identifying the vertices $j, j^{\prime}$.

A set of $\dot{k} \operatorname{arcs}\left(i_{r_{s}}, j_{r_{s}}\right), 1 \leq s \leq k$ is called a $k$-crossing if and only if $i_{r_{1}}<i_{r_{2}}<\cdots<$ $i_{r_{k}}<j_{r_{1}}<j_{r_{2}}<\cdots<j_{r_{k}}$ and $k$-nesting if and only if $i_{r_{1}}<i_{r_{2}}<\cdots<i_{r_{k}}<j_{r_{k}}<$ $j_{r_{k-1}}<\cdots<j_{r_{1}}$. A partial matching is called $k$-noncrossing ( $k$-nonnesting) [4], if it does not contain a $k$-crossing ( $k$-nesting). A tangle is $k$-noncrossing ( $k$-nonnesting) if and only if its inflation is a $k$-noncrossing ( $k$-nonnesting) partial matching [5]. It is interesting to 


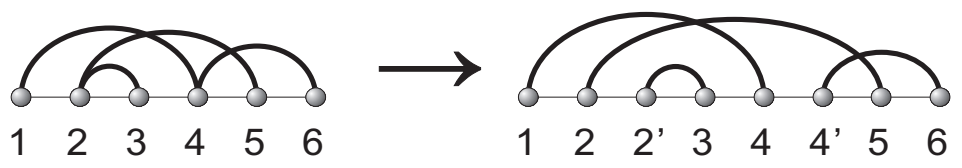

Figure 3: The inflation of a tangled diagram into its corresponding partial matching over 8 vertices.

observe that tangled diagrams are in correspondence with the following types of walks:

Observation 1: The number of $k$-noncrossing tangled diagrams over $[n]$, without isolated points, equals the number of simple lattice walks in $x_{1} \geq x_{2} \geq \cdots \geq x_{k-1} \geq 0$, from the origin back to the origin, taking $n$ days, where at each day the walker can either make one unit step in any (legal) direction, or else feel energetic and make any two consecutive steps (chosen randomly).

Observation 2: The number of $k$-noncrossing tangled diagrams over $[n]$, (allowing isolated points), equals the number of simple lattice walks in $x_{1} \geq x_{2} \geq \cdots \geq x_{k-1} \geq 0$, from the origin back to the origin, taking $n$ days, where at each day the walker can either feel lazy and stay in place, or make one unit step in any (legal) direction, or else feel energetic and make any two consecutive steps (chosen randomly).

These follow easily from the consideration in [5], and are left as amusing exercises for the readers. The paper is organized as follows: in Section 2 we consider enumeration and computation using the holonomic framework [14]. In Section 3 we validate that the formula, proved in Section 2 for $k=2,3,4$, holds for arbitrary $k$.

\section{Efficient enumeration}

Let $t_{k}(n)$ and $\tilde{t}_{k}(n)$ denote the numbers of $k$-noncrossing tangled diagrams with and without isolated points, respectively. Furthermore let $f_{k}(m)$ denote the number of $k$ noncrossing matchings over $m$ vertices or equivalently be the number of ways of walking $n$ steps in $x_{1} \geq x_{2} \geq \cdots \geq x_{k-1} \geq 0$, from the origin back to the origin. Then, as shown in [5], $\tilde{t}_{k}(n)$ and $t_{k}(n)$ are given by:

$$
\tilde{t}_{k}(n)=\sum_{i=0}^{n}\left(\begin{array}{l}
n \\
i
\end{array}\right) f_{k}(2 n-i) \quad \text { and } \quad t_{k}(n)=\sum_{i=0}^{n}\left(\begin{array}{c}
n \\
i
\end{array}\right) \tilde{t}_{k}(n-i) .
$$

As for $f_{k}(n)$, Grabiner and Magyar proved an explicit determinant formula, [9] (see also [4], eq. 9) that expresses the exponential generating function of $f_{k}(n)$, for fixed $k$, as a $(k-1) \times(k-1)$ determinant

$$
\sum_{n \geq 0} f_{k}(2 n) \cdot \frac{x^{2 n}}{(2 n) !}=\left.\operatorname{det}\left[I_{i-j}(2 x)-I_{i+j}(2 x)\right]\right|_{i, j=1} ^{k-1},
$$


where $I_{m}(2 x)$ is the hyperbolic Bessel function:

$$
I_{m}(2 x)=\sum_{j=0}^{\infty} \frac{x^{m+2 j}}{j !(m+j) !} .
$$

Recall that a formal power series $G(x)$ is $D$-finite if it satisfies a linear differential equation with polynomial coefficients. For any $m$ the hyperbolic Bessel functions are $D$-finite [11], which is also called $P$-finite in [14]. By general considerations, that we omit here, it is easy to establish a priori bounds for the order of the recurrence, and for the degrees of its polynomial coefficients, any empirically derived recurrence (using the command listtorec in the Salvy-Zimmerman Maple package gfun, that we adapted to our own needs in our own package TANGLE), is ipso facto rigorous. We derived explicit recurrences for $k=2,3,4$, and they can be found in the webpage of this article. Also, once recurrences are found, they are very efficient in extending the counting sequences. In the same page one can find the sequences for $T_{k}(n)$ for $1 \leq n \leq 1000$, for $k=2,3,4$, and the sequences for $1 \leq n \leq 50$ for $k=5,6$ (using a variant of the Grabiner-Magyar formula implemented in our Maple package TANGLE ).

Once the existence of a recursion is established, we can, for $k=2,3$, 4, employ the Birkhoff-Tritzinsky theory $[2,13]$ and non-rigorous "series analysis" due to Zinn-Justin $[3,15]$. This allows us to safely conjecture that, for any fixed $k$, we have the following asymptotic formula:

$$
t_{k}(n) \sim c_{k} \cdot n^{-\left((k-1)^{2}+(k-1) / 2\right)}\left(4(k-1)^{2}+2(k-1)+1\right)^{n} \quad \text { for some } c_{k}>0 .
$$

In the next Section we shall prove eq. (2.4) for arbitrary $k$.

\section{Asymptotics of tangled diagrams for arbitrary $k$}

In Lemma 3.1 we relate the generating functions of $k$-noncrossing tangled diagrams $T_{k}(z)=\sum_{n} t_{k}(n) z^{n}$ and $k$-noncrossing matchings [4] $F_{k}(z)=\sum_{n} f_{k}(2 n) z^{2 n}$. The functional equation derived will be instrumental to prove eq. (2.4) for arbitrary $k$. For this purpose we shall employ Cauchy's integral formula: let $D$ be a simply connected domain and let $C$ be a simple closed positively oriented contour that lies in $D$. If $f$ is analytic inside $C$ and on $C$, except at the points $z_{1}, z_{2}, \ldots, z_{n}$ that the interior of $C$, then we have Cauchy's integral formula

$$
\int_{C} f(z) d z=2 \pi i \sum_{k=1}^{n} \operatorname{Res}\left[f, z_{k}\right] .
$$

In particular, if $f$ has a simple pole at $z_{0}$, then $\operatorname{Res}\left[f, z_{0}\right]=\lim _{z \rightarrow z_{0}}\left(z-z_{0}\right) f(z)$.

Lemma 3.1. Let $k \in \mathbb{N}, k \geq 2$ and $|z|<2$. Then we have

$$
T_{k}\left(\frac{z^{2}}{1+z+z^{2}}\right)=\frac{1+z+z^{2}}{z+2} F_{k}(z) .
$$


Proof. The relation between the number of $k$-noncrossing tangled diagrams, $t_{k}(n)$ and $k$-noncrossing matchings, $f_{k}(2 m)$ given in eq. (2.1) implies

$$
t_{k}(n)=\sum_{r, \ell}\left(\begin{array}{l}
n \\
r
\end{array}\right)\left(\begin{array}{c}
n-r \\
\ell
\end{array}\right) f_{k}(2 n-2 r-\ell) .
$$

Expressing the combinatorial terms by contour integrals we obtain

$$
\begin{aligned}
&\left(\begin{array}{c}
n \\
r
\end{array}\right)=\frac{1}{2 \pi i} \oint_{|u|=\alpha}(1+u)^{n} u^{-r-1} d u \\
& f_{k}(2 n-2 r-\ell)=\frac{1}{2 \pi i} \oint_{|z|=\beta_{3}} F_{k}(z) z^{-(2 n-2 r-\ell)-1} d z \\
& t_{k}(n)=\sum_{r, \ell}\left(\begin{array}{c}
n \\
r
\end{array}\right)\left(\begin{array}{c}
n-r \\
\ell
\end{array}\right) f_{k}(2 n-2 r-\ell) \\
&=\frac{1}{(2 \pi i)^{3}} \sum_{r, \ell} \oint_{\begin{array}{l}
|v|=\beta_{1} \\
|z|=\beta_{2} \\
|u|=\beta_{3}
\end{array}}(1+u)^{n} u^{-r-1}(1+v)^{n-r} v^{-\ell-1} \times \\
& F_{k}(z) z^{-(2 n-2 r-\ell)-1} d v d u d z
\end{aligned}
$$

where $\alpha, \beta_{1}, \beta_{2}, \beta_{3}$ are arbitrary small positive numbers. Due to absolute convergence of the series we derive

$$
\begin{array}{r}
t_{k}(n)=\frac{1}{(2 \pi i)^{3}} \sum_{r} \oint_{\begin{array}{l}
|v|=\beta_{1} \\
|z|=\beta_{2} \\
|u|=\beta_{3}
\end{array}}(1+u)^{n} u^{-r-1} F_{k}(z) z^{-2 n+2 r-1}(1+v)^{n-r} v^{-1} \times \\
\sum_{\ell}\left(\frac{z}{v}\right)^{\ell} d v d u d z
\end{array}
$$

which is equivalent to

$$
\begin{aligned}
t_{k}(n)=\frac{1}{(2 \pi i)^{3}} \sum_{r} \oint_{\substack{|u|=\beta_{3} \\
|z|=\beta_{2}}}(1+u)^{n} u^{-r-1} F_{k}(z) z^{-2 n+2 r-1} \times & \\
& \left(\oint_{|v|=\beta_{1}} \frac{(1+v)^{n-r}}{v-z} d v\right) d u d z .
\end{aligned}
$$

Since $v=z$ is the only (simple) pole in the integration domain, eq. (3.1) implies

$$
\oint_{|v|=\beta_{1}} \frac{(1+v)^{n-r}}{v-z} d v=2 \pi i(1+z)^{n-r} .
$$

We accordingly obtain

$$
t_{k}(n)=\frac{1}{(2 \pi i)^{2}} \sum_{r} \oint_{\substack{|u|=\beta_{3} \\|z|=\beta_{2}}}(1+u)^{n} u^{-r-1} F_{k}(z) z^{-2 n+2 r-1}(1+z)^{n-r} d u d z .
$$


Proceeding analogously w.r.t. the summation over $r$ yields

$$
\begin{aligned}
t_{k}(n) & =\frac{1}{(2 \pi i)^{2}} \oint_{\substack{|u|=\beta_{3} \\
|z|=\beta_{2}}}(1+u)^{n} F_{k}(z) z^{-2 n-1}(1+z)^{n} u^{-1} \sum_{r} \frac{z^{2 r}}{u^{r}(1+z)^{r}} d u d z \\
& =\frac{1}{(2 \pi i)^{2}} \oint_{|z|=\beta_{2}} F_{k}(z) z^{-2 n-1}(1+z)^{n}\left(\oint_{|u|=\beta_{3}}(1+u)^{n} \frac{1}{u-\frac{z^{2}}{1+z}} d u\right) d z .
\end{aligned}
$$

Since $u=\frac{z^{2}}{1+z}$ is the only pole in the integration domain, Cauchy's integral formula implies

$$
\oint_{|u|=\beta_{3}}(1+u)^{n} \frac{1}{u-\frac{z^{2}}{1+z}} d u=2 \pi i\left(1+\frac{z^{2}}{1+z}\right)^{n} .
$$

We finally compute

$$
\begin{aligned}
t_{k}(n) & =\frac{1}{2 \pi i} \oint_{|z|=\beta_{2}} F_{k}(z) z^{-1} z^{-2 n}(1+z)^{n}\left(1+\frac{z^{2}}{1+z}\right)^{n} d z \\
& =\frac{1}{2 \pi i} \oint_{|z|=\beta_{2}} F_{k}(z) z^{-1}\left(\frac{1+z+z^{2}}{z^{2}}\right)^{n} d z \\
& =\frac{1}{2 \pi i} \oint_{|z|=\beta_{2}} \frac{1+z+z^{2}}{z+2} F_{k}(z)\left(\frac{z^{2}}{1+z+z^{2}}\right)^{-n-1} d\left(\frac{z^{2}}{1+z+z^{2}}\right)
\end{aligned}
$$

and the lemma follows from Cauchy's integral formula

$$
T_{k}\left(\frac{z^{2}}{1+z+z^{2}}\right)=\frac{1+z+z^{2}}{z+2} F_{k}(z) .
$$

Theorem 3.2. For arbitrary $k \in \mathbb{N}, k \geq 2$ the number of tangled diagrams is asymptotically given by

$$
t_{k}(n) \sim c_{k} n^{-\left((k-1)^{2}+\frac{k-1}{2}\right)}\left(4(k-1)^{2}+2(k-1)+1\right)^{n} \quad \text { where } c_{k}>0 .
$$

Proof. According to $[11,14], F_{k}(x)=\sum_{n} f_{k}(2 n) x^{2 n}$ and $T_{k}(x)$ are both $D$-finite. Therefore both have a respective singular expansion [7]. We consider the following asymptotic formula for $f_{k}(2 n)$ [10]: for arbitrary $k \geq 2$

$$
f_{k}(2 n) \sim n^{-\left((k-1)^{2}+\frac{k-1}{2}\right)}(2(k-1))^{2 n} .
$$

Eq. (3.6) allows us to make two observations. First $F_{k}(x)$ has the positive, real, dominant singularity, $\rho_{k}=(2(k-1))^{-1}$ and secondly, in view of the subexponential factor $n^{-\left((k-1)^{2}+\frac{k-1}{2}\right)}$ :

$$
F_{k}(z)=O\left(\left(z-\rho_{k}\right)^{\left((k-1)^{2}+\frac{k-1}{2}\right)-1}\right), \quad \text { as } z \rightarrow \rho_{k} .
$$


According to Lemma 3.1 we have

$$
T_{k}\left(\frac{z^{2}}{z^{2}+z+1}\right)=\frac{z^{2}+z+1}{z+2} F_{k}(z),
$$

where $|z| \leq \rho_{k} \leq \frac{1}{2}+\epsilon, \epsilon>0$ is arbitrarily small and the function

$$
\vartheta(z)=\frac{z^{2}}{z^{2}+z+1}
$$

is regular at $z=\rho_{k}$. Since the composition $H(\eta(z))$ of a $D$-finite function $H$ and a rational function $\eta$, where $\eta(0)=0$ is $D$-finite [11], the functions $T_{k}(\vartheta(z))$ and $F_{k}(z)$ have singular expansions. Eq. (3.8) and eq. (3.6) imply using Bender's method $\left(F_{k}(z)\right.$ satisfies the "ratio test") [1]

$$
\left[z^{n}\right] T_{k}(\vartheta(z)) \sim \frac{\rho_{k}^{2}+\rho_{k}+1}{\rho_{k}+2}\left[z^{n}\right] F_{k}(z) \sim \frac{\rho_{k}^{2}+\rho_{k}+1}{\rho_{k}+2} n^{-\left((k-1)^{2}+\frac{k-1}{2}\right)}\left(\rho_{k}^{-1}\right)^{2 n} .
$$

Eq. (3.9) implies that

$$
\tau_{k}=\frac{\rho_{k}^{2}}{\rho_{k}^{2}+\rho_{k}+1}
$$

is the positive, real, dominant singularity of $T_{k}(z)$. Indeed, Pringsheim's Theorem [12] guarantees the existence of a positive, real, dominant singularity of $T_{k}(z)$, denoted by $\tau_{k}$. For $0 \leq x \leq 1$ the mapping $x \mapsto \vartheta(x)$ is strictly increasing and continuous, whence $\tau_{k}=\vartheta(\zeta)$ for some $0<\zeta \leq 1$. In view of eq. (3.8), $\zeta$ is the dominant, positive, real singularity of $F_{k}(z)$, i.e. $\zeta=\rho_{k}$. Accordingly,

$$
\left[z^{n}\right] T_{k}(z) \sim \theta(n)\left(\frac{\rho_{k}^{2}}{\rho_{k}^{2}+\rho_{k}+1}\right)^{n} .
$$

We shall proceed by analyzing $T_{k}(z)$ at dominant singularities. We observe that any dominant singularity $v$ can be written as $v=\vartheta(\zeta)$. Let $S_{T_{k}}(z-\vartheta(\zeta))$ denote the singular expansion of $T_{k}(z)$ at $v=\vartheta(\zeta)$. Since $\vartheta(z)$ is regular at $\zeta, T_{k}(\vartheta(z))$ we have the supercritical case of singularity analysis [7]: given $\psi(\phi(z)), \phi$ being regular at the singularity of $\psi$, the singularity-type of the composition is that of $\psi$. Indeed, we have

$$
\begin{aligned}
T_{k}(\vartheta(z)) & =O\left(S_{T_{k}}(\vartheta(z)-\vartheta(\zeta))\right) & & \text { as } \vartheta(z) \rightarrow \vartheta(\zeta) . \\
& =O\left(S_{T_{k}}(z-\zeta)\right) & & \text { as } z \rightarrow \zeta .
\end{aligned}
$$

Eq. (3.8) provides the following interpretation for $T_{k}(\vartheta(z))$ at $z=\zeta$ :

$$
T_{k}(\vartheta(z))=O\left(F_{k}(z)\right) \quad \text { as } z \rightarrow \zeta,
$$

from which we can conclude that $T_{k}(z)$ has at $v=\vartheta(\zeta)$ exactly the same subexponential factors as $F_{k}(z)$ at $\zeta$. We next prove that $\tau_{k}$ is the unique dominant singularity of $T_{k}(z)$. Suppose $v=\vartheta(\zeta)$ is an additional dominant singularity of $T_{k}(z)$. The key observation is

$$
\forall \zeta \in \mathbb{C} \backslash \mathbb{R} ; \quad \vartheta(\zeta)=\tau_{k} \quad \Longrightarrow \quad|\zeta|<\rho_{k} .
$$


Eq. (3.11) implies that if $v$ exists then $\zeta$ is a singularity of $F_{k}(z)$ of modulus strictly smaller than $\rho_{k}$, which is impossible. Therefore $\tau_{k}$ is unique and we derive

$$
\left[z^{n}\right] T_{k}(z) \sim c_{k} n^{-\left((k-1)^{2}+\frac{k-1}{2}\right)}\left(\frac{\rho_{k}^{2}}{\rho_{k}^{2}+\rho_{k}+1}\right)^{n} \quad \text { for some } c_{k}>0
$$

and the theorem follows.

Acknowledgments. We are grateful to Emma Y. Jin for helpful discussions. This work was supported by the 973 Project, the PCSIRT Project of the Ministry of Education, the Ministry of Science and Technology, and the National Science Foundation of China. The fourth author is supported in part by the USA National Science Foundation.

\section{References}

[1] E.A. Bender, Asymptotic methods in enumeration, SIAM Rev, 16 (1974), 485-515.

[2] G.D. Birkhoff and W. J. Trjitzinsky, Analytic theory of singular difference equations, Acta Math., 60 (1932), 1-89.

[3] E. Brezin and J. Zinn-Justin, J. Phys. (Paris), 40 L511 (1979).

[4] W.Y.C. Chen, E.Y.P. Deng, R.R.X. Du, R.P. Stanley and C.H. Yan, Crossings and nestings of matchings and partitions, Trans. Amer. Math. Soc., 359 (2007), 15551575.

[5] W.Y.C. Chen, J. Qin and C.M. Reidys, Crossings and Nestings of tangled diagrams, Electron. J. Combin., 15(1) (2008), \#R86.

[6] G.P. Egorychev, Integral Representation and the computation of combinatorial sums, Translations of Mathematical Monographs, Vol.59, Amer. Math. Soc., 1984.

[7] P. Flajolet and R. Sedgewick, Analytic Combinatorics, Cambridge University Press, 2008.

[8] I. Gessel and D. Zeilberger, Random Walk in a Weyl chamber, Proc. Amer. Math. Soc., 115 (1992), 27-31.

[9] D. Grabiner and P. Magyar, Random Walks in a Weyl Chamber and the decomposition of tensor powers, J. Alg. Combin., 2 (1993), 239-260.

[10] E.Y. Jin, C.M. Reidys and R.R. Wang, Asympotic enumeration of $k$-noncrossing matchings, (2008), Submitted.

[11] R. Stanley, Differentiably Finite Power Series, Europ. J. Combin., 1 (1980), 175-188.

[12] E.C. Titchmarsh, The Theory of Functions, Oxford University Press, London, 1939.

[13] J. Wimp and D. Zeilberger, Resurrecting the asymptotics of linear recurrences, J. Math. Anal. Appl., 3 (1985), 162-176.

[14] D. Zeilberger, A Holonomic systems approach to special functions identities, J. Comput. Appl. Math., 32 (1990), 321-368.

[15] J. Zinn-Justin, J. Phys. (Paris), 42, (1981), 783. 